\title{
Targeted Delivery of Paclitaxel in Liver Cancer Using Hyaluronic Acid Functionalized Mesoporous Hollow Alumina Nanoparticles
}

\author{
Yu Gao $\mathbb{D},{ }^{1}$ Lili Hu, ${ }^{2}$ Ying Liu, ${ }^{2}$ Xiaoyan $X u,{ }^{2}$ and Chao Wu $\mathbb{D}^{2}$ \\ ${ }^{1}$ Department of Medical Oncology, The First Affiliated Hospital of Jinzhou Medical University, No.2, The Fifth Section of Renmin Street, \\ Guta District, Jinzhou, Liaoning Province 121001, China \\ ${ }^{2}$ Department of Pharmaceutics, Jinzhou Medical University, 40 Songpo Road, Linghe District, \\ Jinzhou, Liaoning Province 121001, China
}

Correspondence should be addressed to Chao Wu; wuchao27@126.com

Received 14 December 2018; Revised 26 February 2019; Accepted 24 March 2019; Published 15 April 2019

Academic Editor: Sanyog Jain

Copyright (C) 2019 Yu Gao et al. This is an open access article distributed under the Creative Commons Attribution License, which permits unrestricted use, distribution, and reproduction in any medium, provided the original work is properly cited.

Hyaluronic acid functionalized mesoporous hollow alumina nanoparticles (HMHA) were used as a tumor-targeted delivery carrier for liver cancer therapy. Paclitaxel (PAC) incorporated in the carrier by the adsorption method was analyzed by X-ray diffraction and differential scanning calorimetry. PAC was found to be in an amorphous state. The hyaluronic acid coated on the surface of mesoporous hollow alumina nanoparticles (MHA) regulated the drug release rate and the loaded samples obtained a sustained drug release. In vitro experiments demonstrated that paclitaxel-hyaluronic acid functionalized mesoporous hollow alumina nanoparticles (PAC-HMHA) had a high cellular uptake, which increased the drug level in tumor tissues and was beneficial to promote apoptosis. An in vivo tumor inhibition rate study demonstrated that PAC-HMHA $(64.633 \pm 4.389 \%)$ had a better antitumor effect than that of paclitaxel-mesoporous alumina nanoparticles (PAC-MHA, 56.019 $\pm 6.207 \%$ ) and pure PAC (25.593 \pm 4.115\%). Therefore it can be concluded that PAC-HMHA are a prospective tumor-targeted delivery medium and can be useful for future cancer therapy.

\section{Introduction}

Liver cancer is a lethal disease with high incidence and requires particular attention. Traditional chemotherapy, with high cytotoxicity in various tissues, is perplexing. Currently, novel nanodrug delivery systems have been studied in order to obtain a better therapy for liver cancer and these systems include polymeric nanoparticles [1, 2], liposomes [3, 4], and inorganic material nanoparticles [5-8]. These forms can improve the drug circulation time in the blood and passively target tumor tissues, due to the enhanced permeability and retention effect (EPR). Although passive targeting reduces the side effects of chemotherapeutic drugs, the drug concentration in the tumor cells still is insufficient. For effective targeting, various liver-targeting ligands have been grafted to the surface of the carriers to overcome the problem of low target efficiency. Commonly used ligands include folic acid, galactose, protein, hyaluronic acid (HA), and glycyrrhetinic acid [9-13]. Ligand-modified nanodrug delivery systems can enter tumor tissues by receptor-mediated endocytosis and obtain the optimum antitumor effect.

Recently, HA with high targeting efficiency, good biocompatibility, good biodegradability, and nontoxicity can bind specifically with cluster determinant 44 (CD44) receptors on the hepatoma cell membrane, which is widely applied in the functionalization of carrier materials. Moreover, with the development of nanomaterials, metallic oxides excite researchers for use as antitumor drug carriers, such as zinc oxide nanoparticles [14], copper oxide nanowires [15], alumina nanoparticles [16], and ferric oxide nanoparticles [17]. Their nanostructure characteristics show unique potential as a carrier material. Among them, alumina, with extensive biological application potential, attracts our attention [18-22]. Zhao J. et al. made Vx3-functionalized alumina nanoparticle-loaded ubiquitinated proteins for improving cancer immunotherapy [23]. Xifreperez E. et al. applied bovine serum albumin- (BSA-) functionalized porous alumina particles as a carrier and investigated the early diagnosis 
and targeted treatment for HepG2 tumor cells [24]. Wang Y. et al. prepared anodic alumina nanotubes loaded with the proapoptotic protein apo2L/TRAIL for use as a potential drug carrier for human breast cancer therapy [25]. Owing to good performance of alumina materials, it is a suitable antitumor vector.

In this study, mesoporous hollow alumina nanoparticles (MHA) were prepared by the template method and mesoporous carbon nanoparticles were used as the template material. After the carrier was aminated, HA was grafted with the amino group of MHA for better targeting efficiency. The mesoporous hollow structure of MHA was suitable for drug storage. PAC was integrated with HMHA as a first-line chemotherapeutic drug. The inhibitory and antitumor effects of the prepared PAC-HMHA were analyzed in vitro using liver cancer cells and in vivo using tumor-bearing nude mice.

\section{Materials and Methods}

2.1. Materials. Paclitaxel (PAC) was supplied by Tianfeng Biotechnology Company (Xian, China). Tetrapropyl orthosilicate (TPOS), (3-aminopropyl)triethoxysilane (APTES), N-hydroxysuccinimide(NHS), and 1-ethyl-3-(3-dimethylaminopropyl)carbodiimide (EDC) were purchased from Aladdin reagent company. Ethanol, $\mathrm{NH}_{3} \mathrm{H}_{2} \mathrm{O}$, resorcinol, formaldehyde, hydrofluoric acid, $\mathrm{Al}\left(\mathrm{NO}_{3}\right)_{3}$, hyaluronic acid (HA), methyl tert-butyl ether, chloroform, acetonitrile, fetal bovine serum (FBS), RPMI 1640 medium, 3-(4,5-dimethylthiazol2-yl)-2,5-diphenyltetrazolium (MTT), dimethyl sulfoxide (DMSO), propidium iodide (PI), Annexin V-FITC, and trypsin were bought from Beijing Dingguo Changsheng Biotech Co., Ltd. (Beijing, China).

\subsection{Preparation of $H M H A$}

2.2.1. Synthesis of Particles. Mesoporous carbon hollow nanospheres were synthesized according to the previous reports [26]. Briefly, TPOS $(3.46 \mathrm{~mL})$ was dripped into a mixed solution composed of ethanol $(70 \mathrm{~mL}), \mathrm{NH}_{3} \mathrm{H}_{2} \mathrm{O}$ ( $3 \mathrm{~mL})$, and $\mathrm{H}_{2} \mathrm{O}(10 \mathrm{~mL})$ under stirring for $15 \mathrm{~min}$, and then resorcinol $(0.4 \mathrm{~g})$ and formaldehyde $(0.56 \mathrm{~mL})$ were added to the above solution. The reaction lasted $24 \mathrm{~h}$ under stirring. The precipitates obtained by centrifugation were dried at $50^{\circ} \mathrm{C}$ and then calcined at $700^{\circ} \mathrm{C}$ under a $\mathrm{N}_{2}$ atmosphere for $7 \mathrm{~h}$. To remove the silica, $5 \mathrm{wt} \%$ of hydrofluoric acid was used. The obtained product was mesoporous carbon hollow nanospheres (MCHN), of which, $100 \mathrm{mg}$ was dispersed in $10 \mathrm{~mL}$ of $\mathrm{Al}\left(\mathrm{NO}_{3}\right)_{3}$ solution $(1.5 \mathrm{M})$ under stirring. After $24 \mathrm{~h}$, the $\mathrm{MCHN}-\mathrm{Al}\left(\mathrm{NO}_{3}\right)_{3}$ samples obtained by centrifugation were dried at $50^{\circ} \mathrm{C}$ and then calcined at $500^{\circ} \mathrm{C}$ under an air atmosphere for $3 \mathrm{~h}$. The product was mesoporous hollow alumina nanoparticles (MHA). Amino-functionalized MHA $\left(\mathrm{MHA}-\mathrm{NH}_{2}\right)$ was obtained by reacting with APTES [27]. A certain amount of NHS and EDC was added to $70 \mathrm{~mL}$ of HA solution $(2 \mathrm{mg} / \mathrm{mL})$ under stirring for activating carboxyl of HA. Two hundred milligrams of amino-functionalized MHA was added to the above HA solution. The system was stirred for $12 \mathrm{~h}$. The product (HMHA) obtained by centrifugation was dehydrated with ethanol and then dried under a vacuum.
2.3. Drug Loading. The adsorption equilibrium method was used to load PAC into HMHA [28]. Briefly, $100 \mathrm{mg}$ of HMHA was dispersed in $100 \mathrm{mg} / \mathrm{mL}$ of PAC chloroform solution under stirring for $5 \mathrm{~h}$. The obtained samples by centrifugation were dried under a vacuum. The drug loading of MHA is the same as the above process. Paclitaxel-mesoporous alumina nanoparticles (PAC-MHA) were used for comparison. Highperformance liquid chromatography (HPLC, LC-2030, Shimadzu, Japan) was applied to determine the drug loading. The mobile phase was a solution composed of acetonitrile and water with a ratio of 50:50 (v/v). The drug loading (DL) of HMHA was obtained by the following equation.

$$
\text { DL }(\%)=\frac{\text { PAC amount in PAC-HMHA }}{\text { PAC-HMHA amount }}
$$

2.4. Characterization. Transmission electron microscopy (TEM, Tecnai G2F30, FEI, USA) was employed to observe the morphological structure characteristics of carriers. The HA content in HMHA was estimated by thermogravimetric analysis (TGA). The equipment used was a TGA-50 instrument (Shimadzu, Japan). The current state of the drug was observed under nitrogen using a differential scanning calorimeter (DSC-60, Shimadzu, Inc. Japan). The X-ray diffraction (XRD) patterns of the samples were obtained using an X-ray diffractometer (Rigaku Denki, Japan) with a range $(2 \theta)$ from $3^{\circ} \mathrm{C}$ to $60^{\circ} \mathrm{C}$. Fourier transform infrared spectroscopy (FTIR) was performed by FTIR spectrometry (Bruker IFS 55, Switzerland).

2.5. Drug Release. The release test was performed by the paddle method (RC806D, Tianda Tianfa, China). The samples (equivalent to $3 \mathrm{mg}$ PAC) were dispersed in $400 \mathrm{~mL}$ of PBS release medium with $\mathrm{pH}$ 7.4. Drug release medium satisfied sink conditions. The speed and temperature were $100 \mathrm{rpm}$ and $37^{\circ} \mathrm{C}$. At $5 \mathrm{~min}, 10 \mathrm{~min}, 15 \mathrm{~min}, 20 \mathrm{~min}, 30 \mathrm{~min}, 45 \mathrm{~min}$, $1 \mathrm{~h}, 2 \mathrm{~h}, 4 \mathrm{~h}, 6 \mathrm{~h}, 12 \mathrm{~h}$, and $24 \mathrm{~h}, 2 \mathrm{~mL}$ of release medium was taken and centrifuged at $10000 \mathrm{rpm}$ for $10 \mathrm{~min}$. Then, the PAC concentration was determined by HPLC.

2.6. Cell Viability Assays. The cytotoxicity assays of HMHA and the loaded samples were performed with liver cancer SMMC-7721 cell line. The counted cells were cultured in 96well plates under a $\mathrm{CO}_{2}$ atmosphere. The culture medium used was RPMI 1640 medium containing 10\% FBS. After $24 \mathrm{~h}$, the cells adhered to the wall and the medium was replaced with sample suspensions with the different concentrations, which were composed of samples, RPMI 1640 medium, FBS, and $2 \%$ hypromellose. After $48 \mathrm{~h}$ of cultivation, the sample suspension was removed and $20 \mu \mathrm{L}$ of MTT $(5 \mathrm{mg} / \mathrm{mL})$ solution was added. The cells were further incubated for $4 \mathrm{~h}$. DMSO $(200 \mu \mathrm{L})$ replaced the above medium, and then the absorbance was determined by microplate reader at $492 \mathrm{~nm}$ after 10 min of shaking.

2.7. Apoptosis Assay. Flow cytometry (Becton Dickinson, Zürich, Switzerland) was used to record the cell apoptosis rate. The counted cells were cultured in 6-well plates under a $\mathrm{CO}_{2}$ atmosphere. The cells adhered to the wall and then 


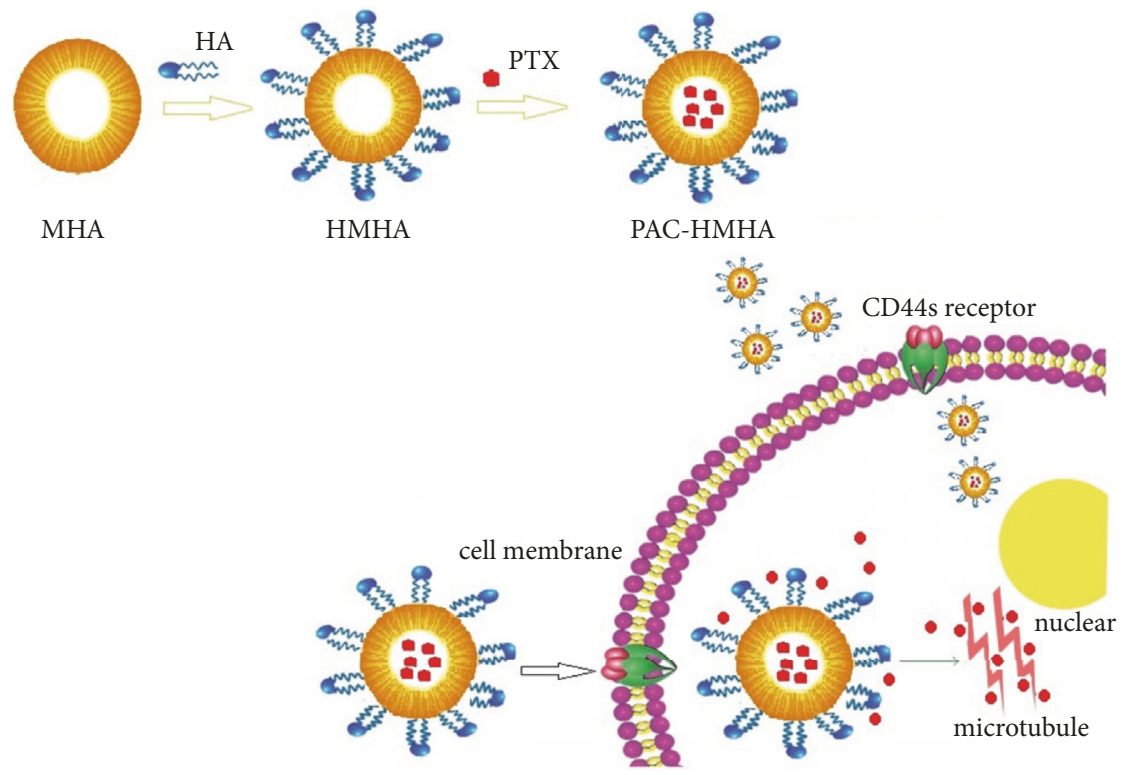

SCHEME 1: Schematic illustration of the preparation process and action mechanism of PAC-HMHA. MHA, mesoporous hollow alumina nanoparticles; HMHA, hyaluronic acid functionalized mesoporous hollow alumina nanoparticles; PAC-HMHA, paclitaxel-hyaluronic acid functionalized mesoporous hollow alumina nanoparticles.

PAC suspensions, PAC-MHA suspensions, and PAC-HMHA suspensions (correspond to $10 \mathrm{ng} / \mathrm{mL}$ of PAC concentration) replaced the culture medium. After incubation for $48 \mathrm{~h}$, the cells were digested by trypsin. The above medium was replaced by $500 \mu \mathrm{L}$ of binding buffer, and then the cells were stained using Annexin V-FITC $(5 \mu \mathrm{L})$ and PI $(5 \mu \mathrm{L})$. The apoptotic cells were determined by flow cytometry.

2.8. Cellular Uptake. The fluorescent labeling process of the HMHA was as follows. A certain amount of HMHA was dispersed in an FITC ethanol solution $(2 \mathrm{mg} / \mathrm{mL})$ and the system was stirred for $5 \mathrm{~h}$. The isothiocyanato group of FITC could bind with the amino group on the inner surface of the HMHA channel. The labeling process of MHA with the amino group was the same as that described above. FITC-labeled MHA was used as a contrast. FITC-labeled HMHA was obtained and used to observe the cellular uptake of HMHA. The counted cells were cultured in a confocal culture dish under a $\mathrm{CO}_{2}$ atmosphere for $24 \mathrm{~h}$. FITC-labeled HMHA suspensions (the equivalent of $50 \mu \mathrm{g} / \mathrm{mL}$ PAC) were used to treat the cells for $0.5 \mathrm{~h}, 1 \mathrm{~h}$, and $1.5 \mathrm{~h}$, and then $4 \%$ paraformaldehyde solution fixed the cells. After staining by Hoechst 33342 and rhodamine B, the cells were observed by confocal laser scanning microscopy. The process of FITClabeled MHA was the same as that described above.

In order to further quantify the PAC uptake efficiency, the counted cells were cultured in culture bottles for $24 \mathrm{~h}$. The PAC suspension, PAC-HMHA suspension, and PAC-MHA suspension (the equivalent of $50 \mu \mathrm{g} / \mathrm{mL}$ PAC for PAC-HMHA and PAC-MHA) were used to treat the cells for $0.5 \mathrm{~h}, 1 \mathrm{~h}$, and $1.5 \mathrm{~h}$. After the cells were collected, they were lysed using ultrasonicator to extract the drug. Methyl tert-butyl ether, as the extraction solvent, was evaporated, and then the obtained dried samples were redissolved in the mobile phase. HPLC was used to analyze the drug concentration.

2.9. Antitumor Efficacy. The antitumor efficacy of PACHMHA was checked using a nude mouse model with liver cancer SMMC-7721 cells (Beijing Weitong Lihua Experimental Animal Technology Co. Ltd.). Jin Zhou Medical University Laboratory Animal Ethics Committee (No. 11400700217882) authorized the experiment. In this experiment, $5 \times 10^{4}$ SMMC-7721 cells were injected subcutaneously into the right front legs of nude mice. After the tumor volume reached $50-70 \mathrm{~mm}^{3}, 20$ mice were randomly placed into four groups. Physiological saline, PAC, PAC-MHA, and PAC-HMHA suspension were injected intraperitoneally; the dose was $20 \mathrm{mg} / \mathrm{kg}$ and the time interval was 3 days. A caliper was used to measure the tumor volume before each administration. The equation was $\mathrm{V}=0.5 \times$ length $\times(\text { width })^{2}$. Tumor tissues were excised after 5 dose cycles and fixed with paraformaldehyde. Paraffin-embedded tumor specimens were sliced and stained with hematoxylin and eosin (H\&E). The obtained tumor sections were surveyed by fluorescence microscopy (Leica DMI 4000B, Germany).

2.10. Statistical Analysis. The mean values plus standard deviations were used to show the parameter values. Statistical analysis testing (ANOVA and Bonferroni tests) using SPSS 17.0 software was carried out for all resulting values, and the results were found to be statistically significant.

\section{Results and Discussion}

3.1. Carrier Morphological Structure and Drug Loading. The preparation process and action mechanism of PAC-HMHA are clearly shown in Scheme 1. The hard-template method, 


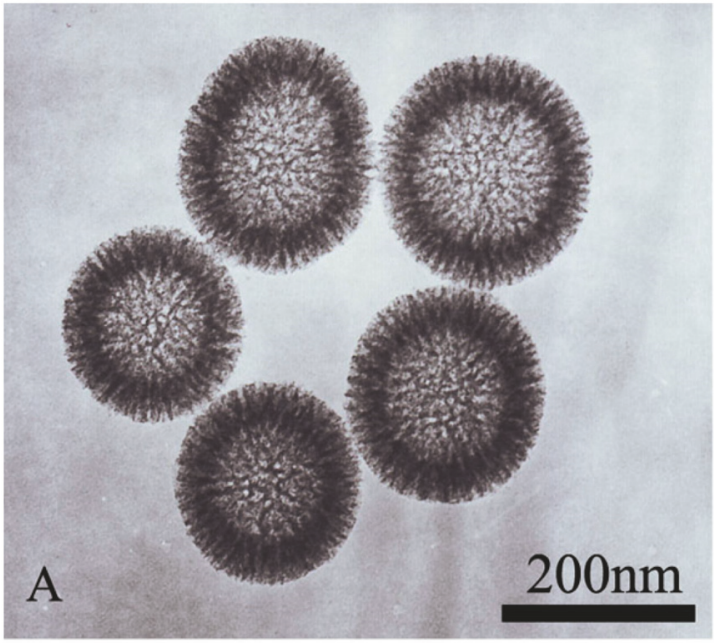

(a)

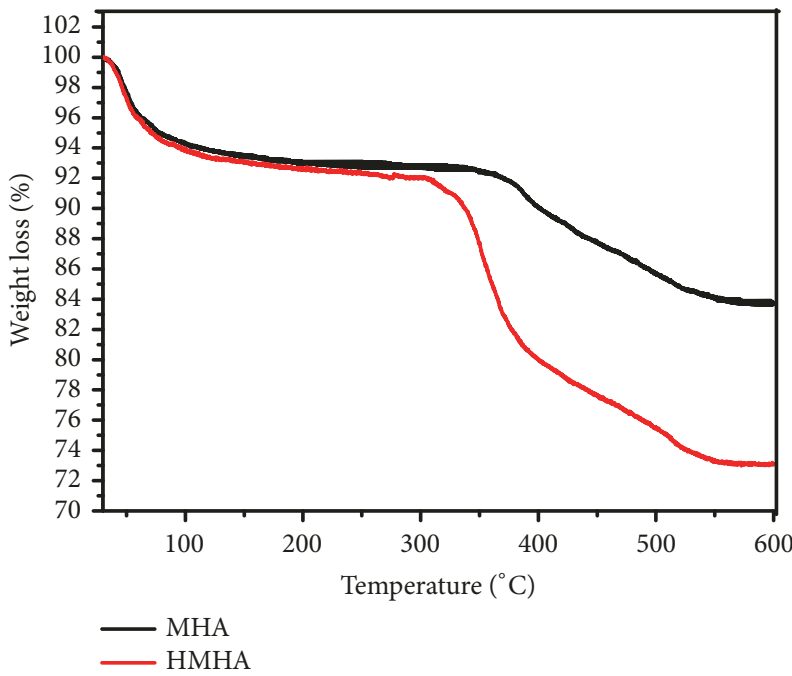

(c)

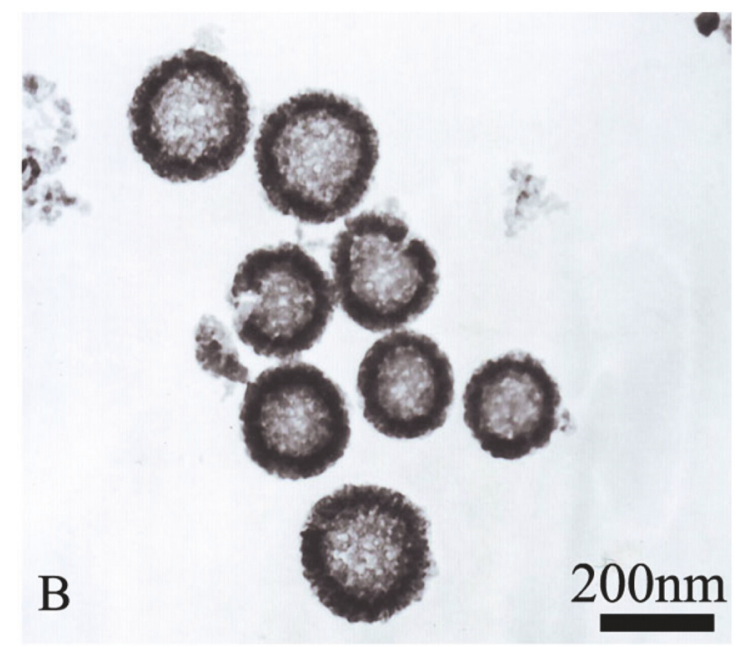

(b)

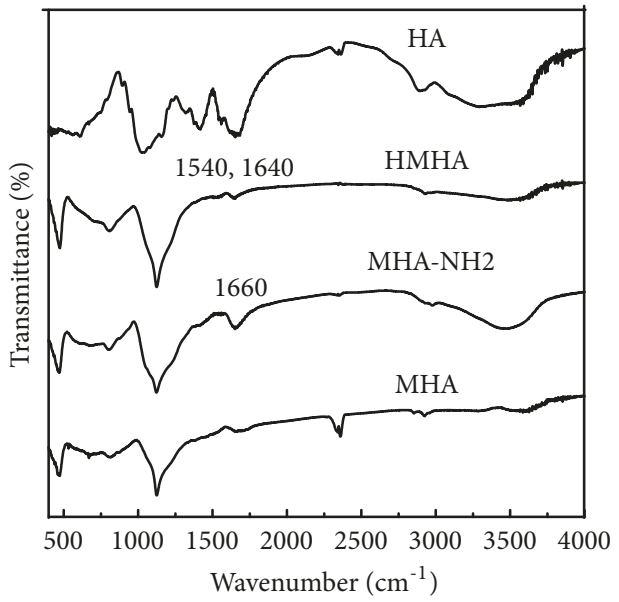

(d)

FIGURE 1: TEM of (a) MCHN and (b) MHA; (c) TGA characterization; (d) FTIR characterization. MCHN, mesoporous carbon hollow nanospheres; MHA, mesoporous hollow alumina nanoparticles; $\mathrm{MHA}-\mathrm{NH}_{2}$, amino-functionalized $\mathrm{MHA}$; $\mathrm{HMHA}$, hyaluronic acid functionalized mesoporous hollow alumina nanoparticles; HA, hyaluronic acid.

with advantages including a simple and scalable process, monodispersed morphology, and adjustable particle size, was used for synthesizing MHA. A mesoporous carbon hollow nanosphere (MCHN) was prepared first as the hard template. Aluminum nitrate was adsorbed into the mesoporous structure of $\mathrm{MCHN}$, and then MHA was obtained by high-temperature calcination followed by modification of the surface of the nanoparticles with the amino group. The carboxyl group of HA covalently cooperated with the amino group on the surface of the MHA. The obtained HMHA was loaded with PAC by the adsorption method. The PAC-HMHA can be efficiently incepted into the cell because HA can be specifically recognized and combined with the CD44 receptor on the surface of the cell membrane [29]. In our study, MCHN and MHA were successfully prepared. As shown in Figure 1(a), the TEM image of MCHN had a mesoporous wall and hollow structure. The particle size was approximately $200 \mathrm{~nm}$. The TEM image of MHA in Figure 1(b) displayed the mesoporous hollow structure, which was successfully replicated from the MCHN. In order to verify the cladding of HA, a TGA diagram (Figure 1(c)) showed that HMHA presented a weight loss of approximately $6 \%$ compared with amino-functionalized MHA (MHA$\mathrm{NH}_{2}$ ), which explained the covered HA amount. The FTIR spectra results in Figure $1(\mathrm{~d})$ indicate that $\mathrm{MHA}-\mathrm{NH}_{2}$ had a $\mathrm{NH}_{2}$ bending vibration peak at $1660 \mathrm{~cm}^{-1}$. In HMHA, $1540 \mathrm{~cm}^{-1}$ (NH in-plane bending vibration) and $1640 \mathrm{~cm}^{-1}$ (amide bond) indicated that the HA successfully bonded with the amino groups. The drug loading of PAC-HMHA was $29.45 \pm 2.16 \%$, whereas that of PAC-MHA was $35.21 \pm$ $1.91 \%$. The pore volume of the carrier decreased due to the coating of $\mathrm{HA}$, which induced a reduction in drug loading. The above results showed that HMHA was suitable for drug storage. 


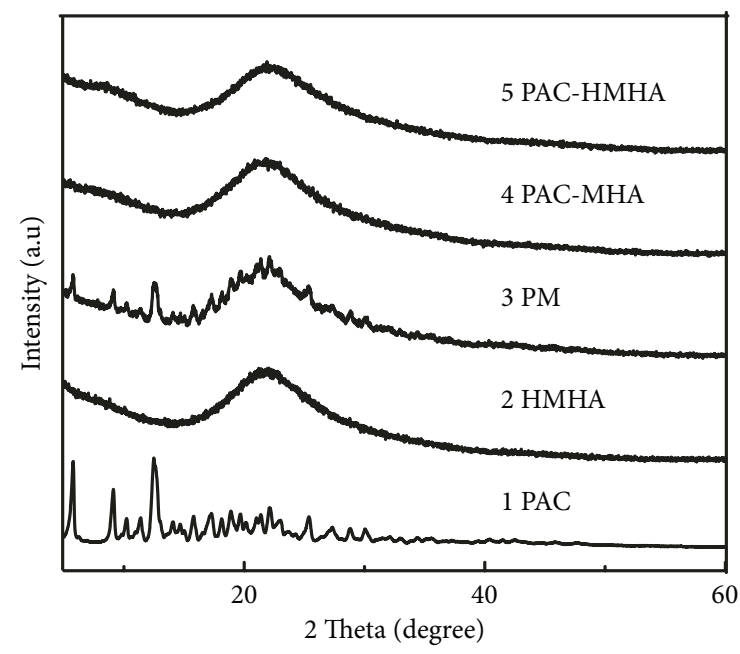

(a)

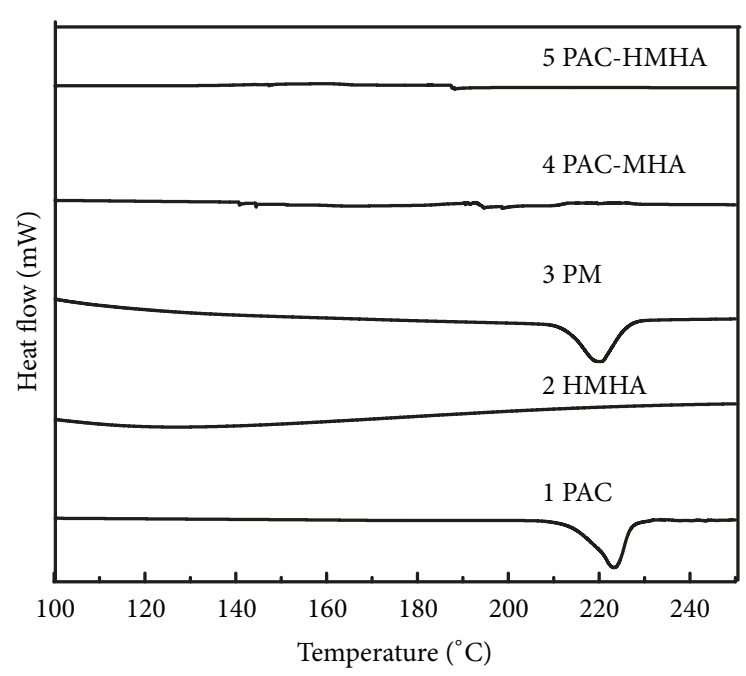

(b)

FIgure 2: The XRD (a) and DSC (b) characterization of PAC, HMHA, PM, PAC-MHA, and PAC-HMHA. PAC, paclitaxel; HMHA, hyaluronic acid functionalized mesoporous hollow alumina nanoparticles; PM, the physical mixture of the drug and HMHA; PAC-MHA, paclitaxel-mesoporous hollow alumina nanoparticles; PAC-HMHA, paclitaxel-hyaluronic acid functionalized mesoporous hollow alumina nanoparticles.

3.2. XRD and DSC Analysis. Figure 2(a) shows the XRD of PAC, HMHA, the physical mixture (PM, the ratio of the drug and HMHA was the same as that of the loaded samples), PAC-MHA, and PAC-HMHA. The XRD results for HMHA was amorphous. PAC and PM displayed the crystalline state of drugs at $5.8^{\circ}$ and $12.5^{\circ}$. By comparison, PAC-MHA and PAC-HMHA did not present the crystalline characteristics of PAC. The above results indicated that PAC in the mesoporous channel of MHA or HMHA was amorphous, which was confirmed by DSC analysis. DSC curves of PAC, HMHA, PM, PAC-MHA, and PAC-HMHA are shown in Figure 2(b). The DSC for HMHA did not show a phase transformation, which indicates that HMHA were amorphous. PAC and PM displayed a phase transformation of the drug at $223^{\circ} \mathrm{C}$. By comparison, PAC-MHA and PAC-HMHA did not present the crystal-phase transformation of PAC at $223^{\circ} \mathrm{C}$. Finally, we identified that PAC was in an amorphous state, which was the direct reason for the improvement in the drug's solubility.

3.3. In Vitro Release. The greatly enhanced dissolution by MHA and regulated release rate by HMHA indicated high drug release in vitro. Therefore, comparative evaluation of the in vitro release of PAC-MHA and PAC-HMHA was carried out in the release test. In Figure 3, compared with $21.59 \pm 1.15 \%$ release of PAC at $1 \mathrm{~h}$, PAC-MHA significantly improved drug dissolution and reached $85.35 \pm 3.25 \%$ at 1h. This indicated that the mesoporous hollow structure of MHA was highly dispersed in PAC and the amorphous state of PAC was the cause of improvement in the drug dissolution. PAC-HMHA exhibited the effect of sustained release, and the dissolution amount reached $89.03 \pm 2.79 \%$ at $24 \mathrm{~h}$. The cladding of HA increased the drug diffusion resistance, which led to the production of the sustained release effect. This could increase the drug absorption and avoid the recrystallization caused by rapid dissolution of PAC.

3.4. In Vitro Cytotoxicity and Apoptosis Analysis. As shown in Figure 4(a), both MHA and HMHA at a maximum concentration of $100 \mu \mathrm{g} / \mathrm{mL}$ showed more than $90 \%$ cell viability, which demonstrated that MHA and HMHA for SMMC-7721 cell had good biosafety. In Figure 4(b), compared with PACMHA and PAC, PAC-HMHA had higher inhibition rate at the $5-250 \mathrm{ng} / \mathrm{mL}$ concentration range for SMMC-7721 cells. At $250 \mathrm{ng} / \mathrm{mL}$ of PAC concentration, cell viability of PAC group was 58.6 $\pm 2.39 \%$. In comparison, PAC-MHA and PACHMHA showed $40.9 \pm 1.83 \%$ and $34.4 \pm 2.04 \%$ cell viability, respectively. The IC50 of PAC, PAC-MHA, and PAC-HMHA were $1022.57 \pm 15.79 \% \mathrm{ng} / \mathrm{mL}, 174.41 \pm 7.48 \% \mathrm{ng} / \mathrm{mL}$, and $77.46 \pm 5.26 \% \mathrm{ng} / \mathrm{mL}$, respectively. All these results illustrated that PAC-HMHA effectively inhibits the proliferation of tumor cells. The nanoscale effect of HMHA and targeting effect of HA could increase the drug accumulation in tumor cells, which leads to high drug concentrations in tumor cells. This was the primary reason for strongly inhibiting cell proliferation [30]. The apoptosis analysis confirmed the above conclusion. PAC-HMHA in Figure 4(c) induced cell early apoptosis in $42.54 \pm 3.19 \%$ of SMMC-7721 cells, in comparison with $10.05 \pm 1.53 \%$ by PAC-MHA and $23.32 \pm$ $1.67 \%$ by PAC at $10 \mathrm{ng} / \mathrm{mL}$ of PAC concentration, indicating that PAC-HMHA effectively promoted apoptosis of SMMC7721 cells. Therefore, HMHA have a targeted effect on SMMC-7721 cells.

3.5. Cellular Uptake Analysis. In order to verify the increased drug accumulation in tumor cells, a cellular uptake test was performed and the results are shown in Figure 5(a). FITClabeled HMHA at $0.5 \mathrm{~h}$ were observed in cells by green 


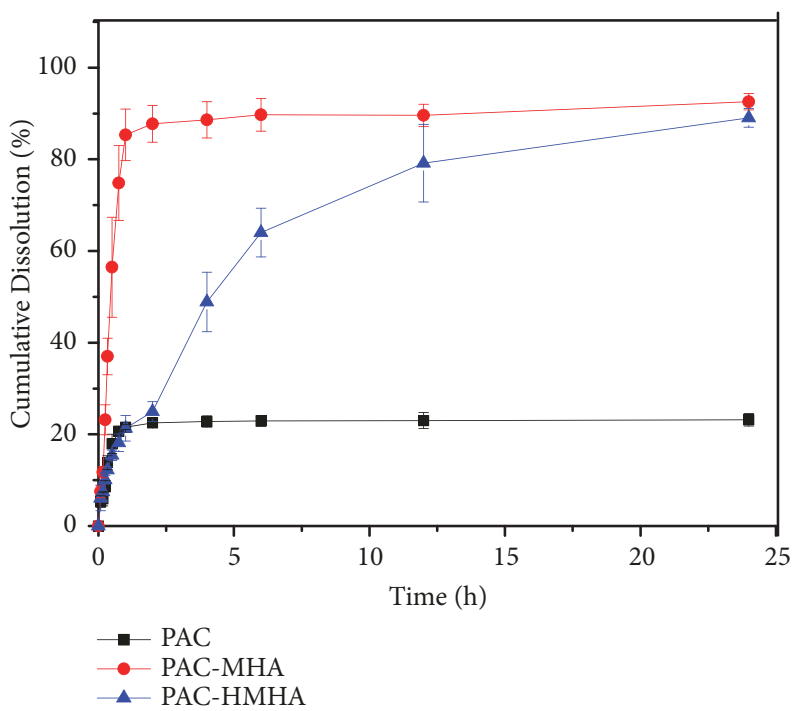

Figure 3: In vitro release curves of PAC, PAC-MHA and PAC-HMHA. Data represented as mean \pm SD ( $n=3$ ). PAC, paclitaxel; PAC-MHA, paclitaxel-mesoporous hollow alumina nanoparticles; PAC-HMHA, paclitaxel-hyaluronic acid functionalized mesoporous hollow alumina nanoparticles.

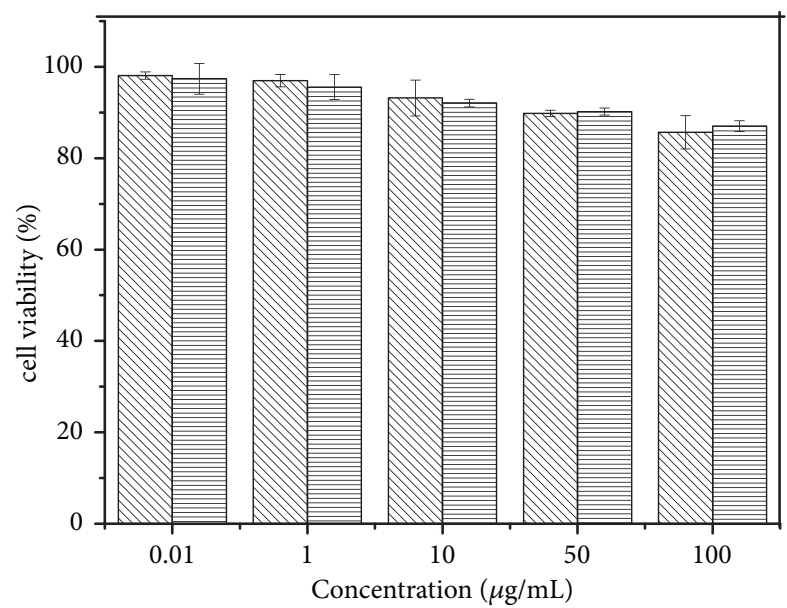

17 MHA

HMHA

(a)

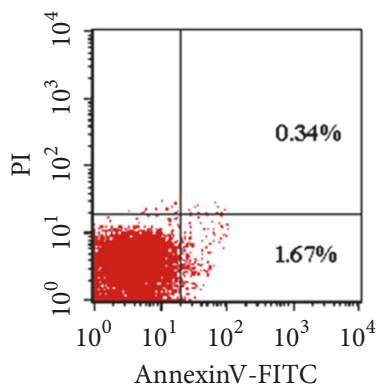

Control

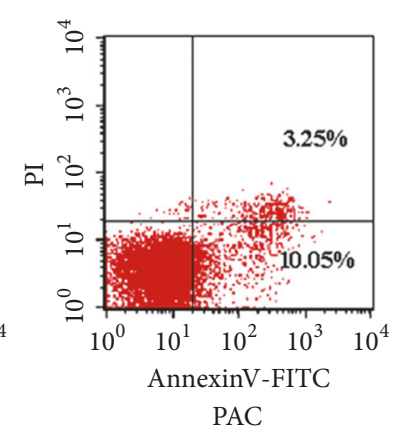

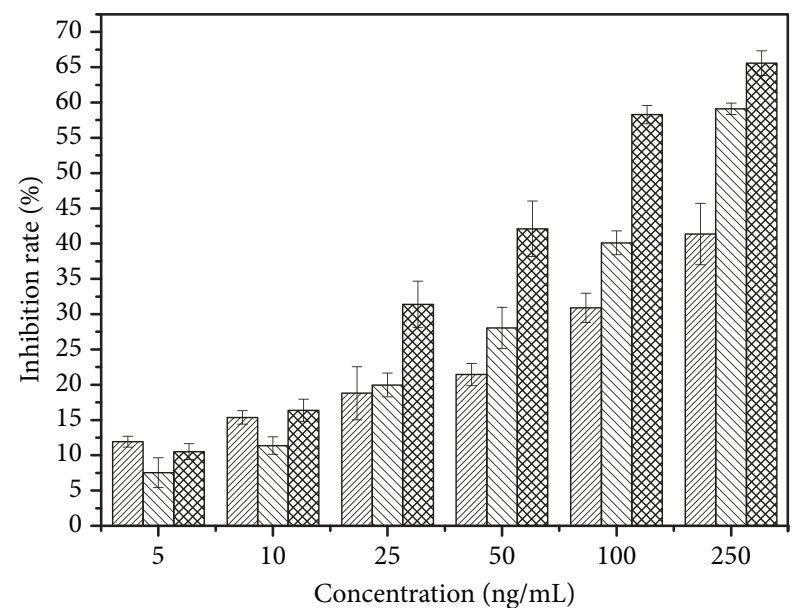

PAC

DIV PAC-MHA

PAC-HMHA

(b)
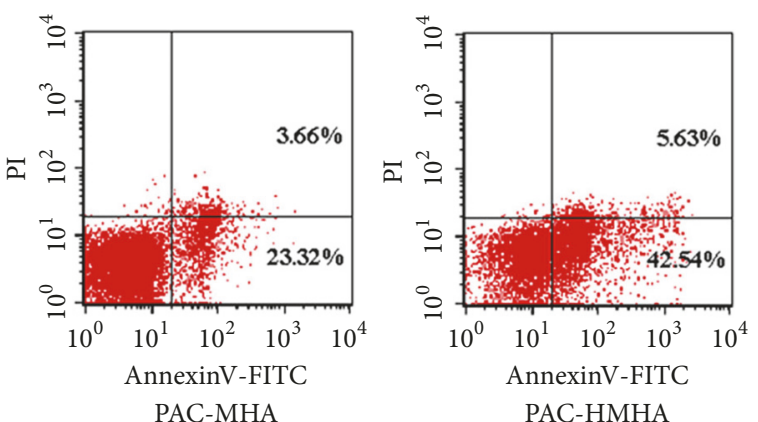

(c)

Figure 4: Cell viability (a) of MHA, HMHA; cell inhibition (b) and apoptosis analysis (c) of PAC, PAC-MHA, and PAC-HMHA. Data represented as mean $\pm \mathrm{SD}(\mathrm{n}=6)$. MHA, mesoporous hollow alumina nanoparticles; HMHA, hyaluronic acid functionalized mesoporous hollow alumina nanoparticles; PAC, paclitaxel; PAC-MHA, paclitaxel-mesoporous hollow alumina nanoparticles; PAC-HMHA, paclitaxelhyaluronic acid functionalized mesoporous hollow alumina nanoparticles. 


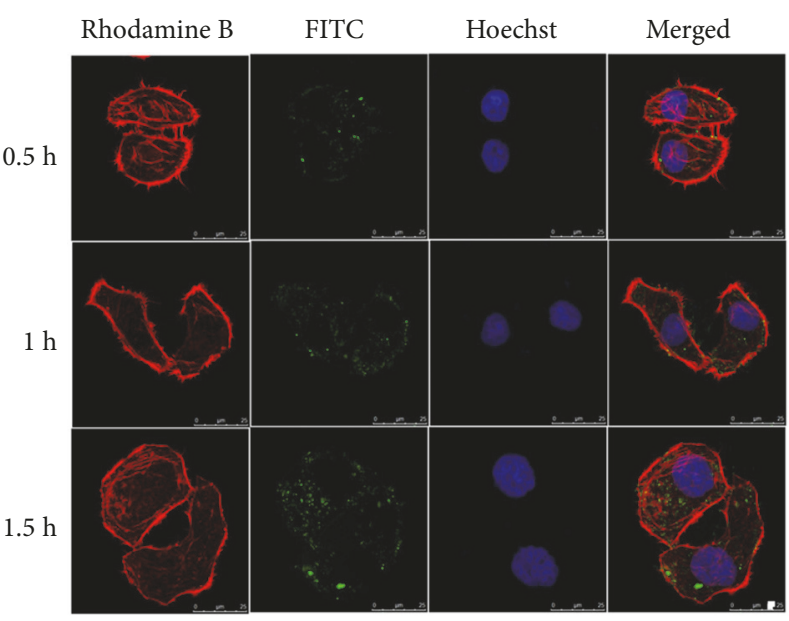

(a)

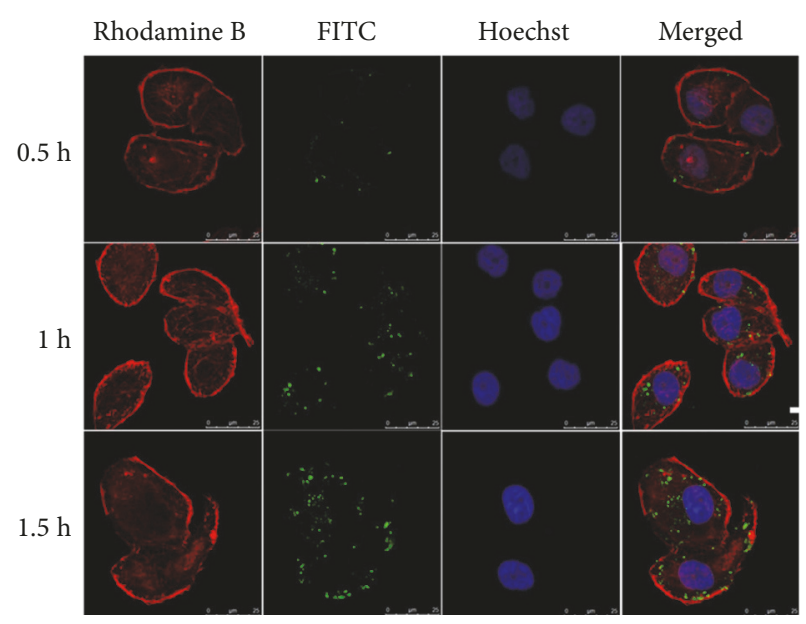

(b)

FIGURE 5: LSCM images of SMMC-7721 cell line treated with FITC-labeled HMHA (a) and FITC-labeled MHA (b) at $0.5 \mathrm{~h}, 1 \mathrm{~h}$, and $1.5 \mathrm{~h}$. MHA, mesoporous hollow alumina nanoparticles; HMHA, hyaluronic acid functionalized mesoporous hollow alumina nanoparticles.

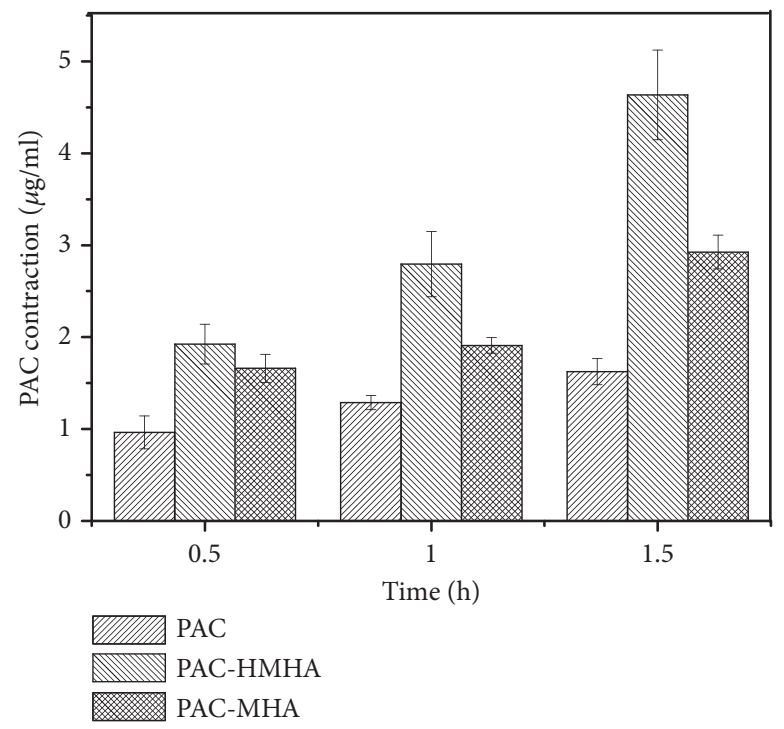

Figure 6: The cellular uptake of PAC, PAC-MHA, and PAC-HMHA from HPLC. PAC, paclitaxel; PAC-MHA, paclitaxel-mesoporous hollow alumina nanoparticles; PAC-HMHA, paclitaxel-hyaluronic acid functionalized mesoporous hollow alumina nanoparticles.

fluorescence, which demonstrated that the carriers were taken up by tumor cells. With increasing incubation time, the intake amount significantly increased according to the changes in the green fluorescence and tumor cells for the uptake of HMHA, indicating time dependence. Moreover, as a comparison, FITC-labeled MHA had a significantly lower intake at the same time, as shown in Figure 5(b). This indicates that the coated HA had an obvious targeting effect. The HPLC results in Figure 6 were consistent with the above results. At $0.5 \mathrm{~h}$, the concentration of PAC in the PACMHA group was $0.963 \pm 0.179 \mu \mathrm{g} / \mathrm{mL}$, compared with 1.662 $\pm 0.154 \mu \mathrm{g} / \mathrm{mL}$ of the PAC group. The PAC-HMHA group had a higher concentration $(1.924 \pm 0.216 \mu \mathrm{g} / \mathrm{mL})$. There is statistical significance $(\mathrm{P}<0.05)$ between $\mathrm{PAC}-\mathrm{HMHA}$ group and PAC-MHA group according to ANOVA and Bonferroni tests. That indicates that the coating of HA enables outstanding targeting of MHA. As time went on, the uptake of PAC also increased significantly in each group, which indicates that the cellular uptake of PAC is time-dependent. The above conclusion will lead to high drug concentration in cells, which will speed up cell apoptosis.

3.6. Antitumor Effect in Solid-Tumor-Bearing Nude Mice. To test whether the antitumor effect in vivo is as good as that in cell experiments, a comparative study of physiological saline, PAC, PAC-MHA, and PAC-HMHA suspension was performed, and the results are shown in Figures 7(a) and 7(b). The PAC-HMHA group $(64.633 \pm 4.389 \%)$ had a better effect at inhibiting the tumor volume than the PAC group (25.593 $\pm 4.115 \%)$ and the PAC-MHA group $(56.019 \pm 6.207 \%)$. The 


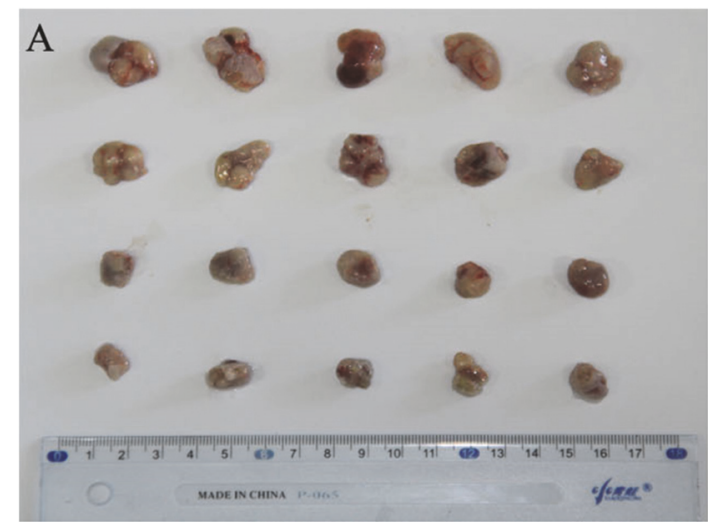

(a)

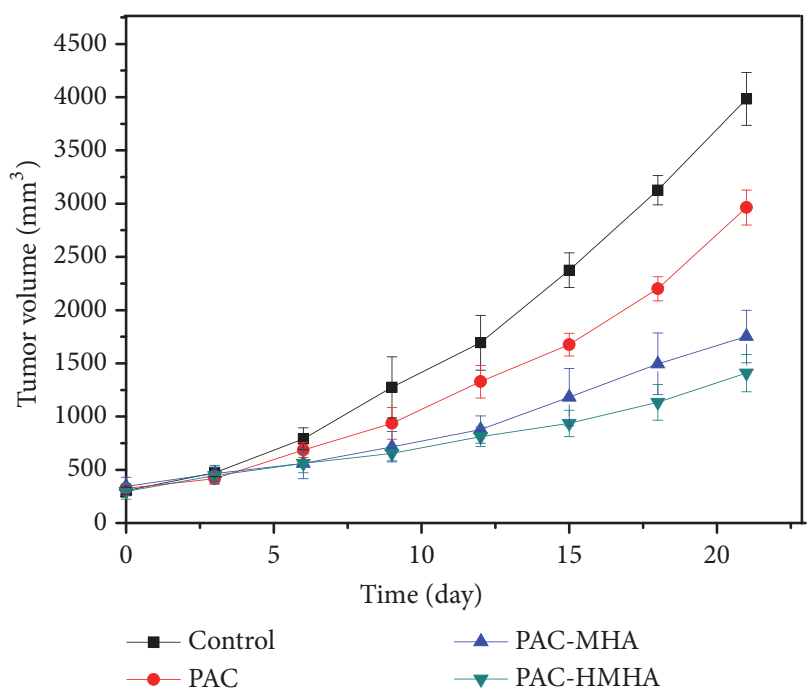

(b)

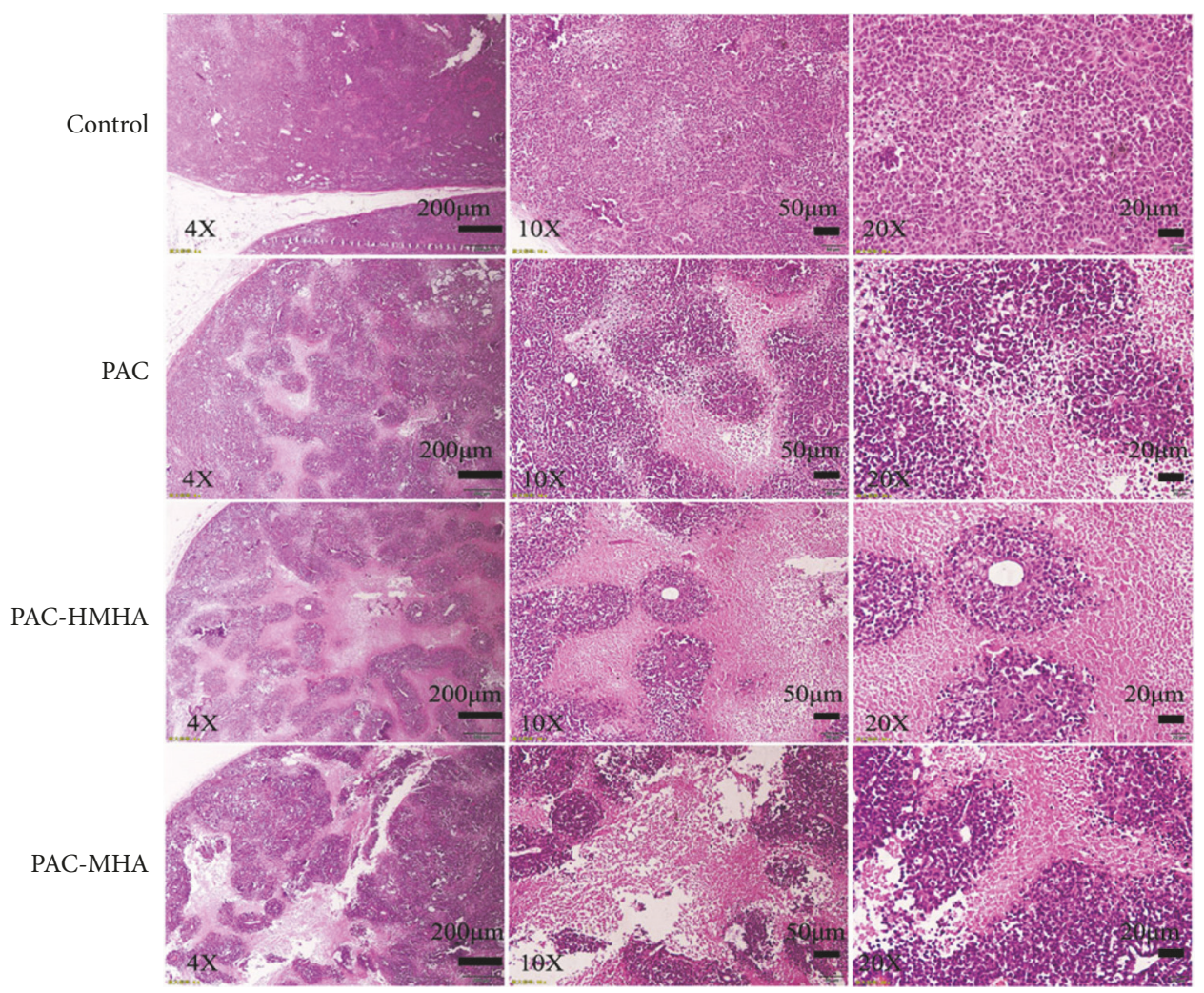

(c)

Figure 7: (a) The tumor tissues images from the tumor-bearing nude mice of physiological saline, PAC, PAC-MHA, and PAC-HMHA groups. (b) The tumor volume curves. (c) Histological examination of excised tumor tissues under different magnification. PAC, paclitaxel; PACMHA, paclitaxel-mesoporous hollow alumina nanoparticles; PAC-HMHA, paclitaxel-hyaluronic acid functionalized mesoporous hollow alumina nanoparticles.

results can be attributed to the accumulation of PAC-HMHA in the tumors due to the nanoscale effect and targeting action of HMHA. The histological photomicrographs of the excised tumor tissues in Figure 7(c) show that the PAC-HMHA group had a more significant necrotic region compared to the PAC group and the PAC-MHA group. The shrinkage and fragmentation of the nuclei was also more visible. In summary, the PAC-HMHA group had a better antiproliferation effect. 


\section{Conclusion}

PAC-HMHA was obtained and used to treat liver cancer. In vitro cell experiments indicated that PAC-HMHA could be ingested by SMMC-7721 cells and prompt cell apoptosis. Compared with PAC and PAC-MHA, PAC-HMHA showed a good tumor-suppression effect in vivo. Overall, this study indicated that HMHA exhibits tumor control ability and is a potential candidate for the development of carrier materials.

\section{Data Availability}

All data generated included in this published article are available from the corresponding author on reasonable request.

\section{Conflicts of Interest}

The authors report no conflicts of interest in this work.

\section{Acknowledgments}

This study was supported by the National Natural Science Foundation of China (no. 81302707), Youth Project of Liaoning Education Department (no. JYTQN201732), the Natural Science Foundation of Liaoning Province (no. 20180550155 and no. 20170540366), Liaoning Province Talent Project Support Programs in Colleges and Universities (no. LJQ2015065), and the Principal Fund-Aohong-Boze-Clinical Medicine Construction Special Fund (no. XZJJ20140205).

\section{References}

[1] Z. Jiang, J. Chi, B. Han, and W. Liu, "Preparation and pharmacological evaluation of norcantharidin-conjugated carboxymethyl chitosan in mice bearing hepatocellular carcinoma," Carbohydrate Polymers, vol. 174, pp. 282-290, 2017.

[2] X. Song, J. You, H. Shao, and C. Yan, "Effects of surface modification of As2O3-loaded PLGA nanoparticles on its antiliver cancer ability: an in vitro and in vivo study," Colloids and Surfaces B: Biointerfaces, vol. 169, pp. 289-297, 2018.

[3] C. Guan, Y. Zhao, Y. Hou, G. Shan, D. Yan, and Y. Liu, "Glycosylated liposomes loading carbon dots for targeted recognition to HepG2 cells," Talanta, vol. 182, pp. 314-323, 2018.

[4] G. Li, D. Liu, E. T. Kimchi et al., "Nanoliposome C6-ceramide increases the anti-tumor immune response and slows growth of liver tumors in mice," Gastroenterology, vol. 154, no. 4, pp. 10241036, 2018.

[5] Z. Wang, Z. Chang, M. Lu et al., "Shape-controlled magnetic mesoporous silica nanoparticles for magnetically-mediated suicide gene therapy of hepatocellular carcinoma," Biomaterials, vol. 154, pp. 147-157, 2018.

[6] J. Wang, Y. Zhou, S. Guo et al., "Cetuximab conjugated and doxorubicin loaded silica nanoparticles for tumor-targeting and tumor microenvironment responsive binary drug delivery of liver cancer therapy," Materials Science and Engineering C: Materials for Biological Applications, vol. 76, pp. 944-950, 2017.

[7] Z. Ji, G. Lin, Q. Lu et al., "Targeted therapy of SMMC-7721 liver cancer in vitro and in vivo with carbon nanotubes based drug delivery system," Journal of Colloid and Interface Science, vol. 365, no. 1, pp. 143-149, 2012.
[8] C. Yu, L. Qian, M. Uttamchandani, L. Li, and S. Q. Yao, "SingleVehicular Delivery of Antagomir and Small Molecules to Inhibit miR-122 Function in Hepatocellular Carcinoma Cells by using "smart" Mesoporous Silica Nanoparticles," Angewandte Chemie International Edition, vol. 54, no. 36, pp. 10574-10578, 2015.

[9] D. M. Anwar, S. N. Khattab, M. W. Helmy et al., "Lactobionic/folate dual-targeted amphiphilic maltodextrin-based micelles for targeted codelivery of sulfasalazine and resveratrol to hepatocellular carcinoma," Bioconjugate Chemistry, vol. 29, no. 9, pp. 3026-3041, 2018.

[10] L. Liu, Z.-M. Zong, Q. Liu et al., "A novel galactose-PEGconjugated biodegradable copolymer is an efficient gene delivery vector for immunotherapy of hepatocellular carcinoma," Biomaterials, vol. 184, pp. 20-30, 2018.

[11] N. A. Hanafy, A. Quarta, R. Di Corato et al., "Hybrid polymericprotein nano-carriers (HPPNC) for targeted delivery of TGF $\beta$ inhibitors to hepatocellular carcinoma cells," Journal of Materials Science: Materials in Medicine, vol. 28, no. 8, p. 120, 2017.

[12] K. S. Kim, W. Hur, S.-J. Park et al., "Bioimaging for targeted delivery of hyaluronic acid derivatives to the livers in cirrhotic mice using quantum dots," ACS Nano, vol. 4, no. 6, pp. 30053014, 2010.

[13] Y. Sun, C. Dai, M. Yin, J. Lu, H. Hu, and D. Chen, "Hepatocellular carcinoma-targeted effect of configurations and groups of glycyrrhetinic acid by evaluation of its derivative-modified liposomes," International Journal of Nanomedicine, vol. 13, pp. 1621-1632, 2018.

[14] M. Zheng, S. Wang, Z. Liu, L. Xie, and Y. Deng, "Development of temozolomide coated nano zinc oxide for reversing the resistance of malignant glioma stem cells," Materials Science and Engineering C: Materials for Biological Applications, vol. 83, pp. 44-50, 2018.

[15] M. Ahir, S. Bhattacharya, S. Karmakar et al., "Tailored-CuOnanowire decorated with folic acid mediated coupling of the mitochondrial-ROS generation and miR425-PTEN axis in furnishing potent anti-cancer activity in human triple negative breast carcinoma cells," Biomaterials, vol. 76, pp. 115-132, 2016.

[16] H. Li, Y. Li, J. Jiao, and H.-M. Hu, "Alpha-alumina nanoparticles induce efficient autophagy-dependent cross-presentation and potent antitumour response," Nature Nanotechnology, vol. 6, no. 10, pp. 645-650, 2010.

[17] J. E. Rosen, L. Chan, D.-B. Shieh, and F. X. Gu, "Iron oxide nanoparticles for targeted cancer imaging and diagnostics," Nanomedicine: Nanotechnology, Biology and Medicine, vol. 8, no. 3, pp. 275-290, 2012.

[18] A. Rajpura, T. N. Board, P. D. Siney et al., "A 28-year clinical and radiological follow-up of alumina ceramic-on-crosslinked polyethylene total hip arthroplasty," The Bone \& Joint Journal, vol. 99B, no. 10, pp. 1286-1289, 2017.

[19] C. C. Camilo, C. A. Silveira, R. S. Faeda, J. M. de Almeida Rollo, B. d. Purquerio, and C. A. Fortulan, "Bone response to porous alumina implants coated with bioactive materials, observed using different characterization techniques," Journal of Applied Biomaterials \& Functional Materials, vol. 15, no. 3, pp. 223-235, 2017.

[20] M. Zhao, Y. Sun, J. Zhang, and Y. Zhang, "Novel translucent and strong submicron alumina ceramics for dental restorations," Journal of Dental Research, vol. 97, no. 3, pp. 289-295, 2018.

[21] S. K. Das, S. Kapoor, H. Yamada, and A. J. Bhattacharyya, "Effects of surface acidity and pore size of mesoporous alumina on degree of loading and controlled release of ibuprofen," 
Microporous and Mesoporous Materials, vol. 118, no. 1-3, pp. 267272, 2009.

[22] S. Kapoor, R. Hegde, and A. J. Bhattacharyya, "Influence of surface chemistry of mesoporous alumina with wide pore distribution on controlled drug release," Journal of Controlled Release, vol. 140, no. 1, pp. 34-39, 2009.

[23] J. Zhao, N. Pan, F. Huang et al., "Vx3-functionalized alumina nanoparticles assisted enrichment of ubiquitinated proteins from cancer cells for enhanced cancer immunotherapy," Bioconjugate Chemistry, vol. 29, no. 3, pp. 786-794, 2018.

[24] E. Xifre-Perez, S. Guaita-Esteruelas, M. Baranowska, J. Pallares, L. Masana, and L. F. Marsal, "In vitro biocompatibility of surface-modified porous alumina particles for HepG2 tumor cells: toward early diagnosis and targeted treatment," ACS Applied Materials \& Interfaces, vol. 7, no. 33, pp. 18600-18608, 2015.

[25] Y. Wang, A. Santos, G. Kaur, A. Evdokiou, and D. Losic, "Structurally engineered anodic alumina nanotubes as nanocarriers for delivery of anticancer therapeutics," Biomaterials, vol. 35, no. 21, pp. 5517-5526, 2014.

[26] S. Purwajanti, H. Zhang, X. Huang et al., "Mesoporous magnesium oxide hollow spheres as superior arsenite adsorbent: synthesis and adsorption behavior," ACS Applied Materials \& Interfaces, vol. 8, no. 38, pp. 25306-25312, 2016.

[27] K. Vimala, K. Shanthi, S. Sundarraj, and S. Kannan, "Synergistic effect of chemo-photothermal for breast cancer therapy using folic acid (FA) modified zinc oxide nanosheet," Journal of Colloid and Interface Science, vol. 488, pp. 92-108, 2017.

[28] J. Xu, Z. Zhao, Y. Hao et al., "Preparation of a novel form of gelatin with a three-dimensional ordered macroporous structure to regulate the release of poorly water-soluble drugs," Journal of Pharmaceutical Sciences, vol. 105, no. 9, pp. 29402948, 2016.

[29] H. Zhao, M. Wu, L. Zhu et al., "Cell-penetrating peptidemodified targeted drug-loaded phase-transformation lipid nanoparticles combined with low-intensity focused ultrasound for precision theranostics against hepatocellular carcinoma," Theranostics, vol. 8, no. 7, pp. 1892-1910, 2018.

[30] P. V. Almeida, M.-A. Shahbazi, E. Mäkilä et al., "Aminemodified hyaluronic acid-functionalized porous silicon nanoparticles for targeting breast cancer tumors," Nanoscale, vol. 6, no. 17, pp. 10377-10387, 2014. 


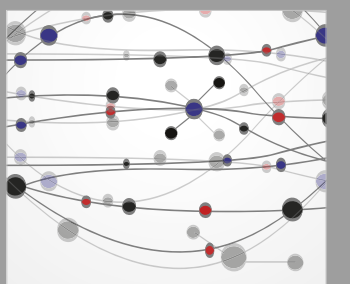

The Scientific World Journal
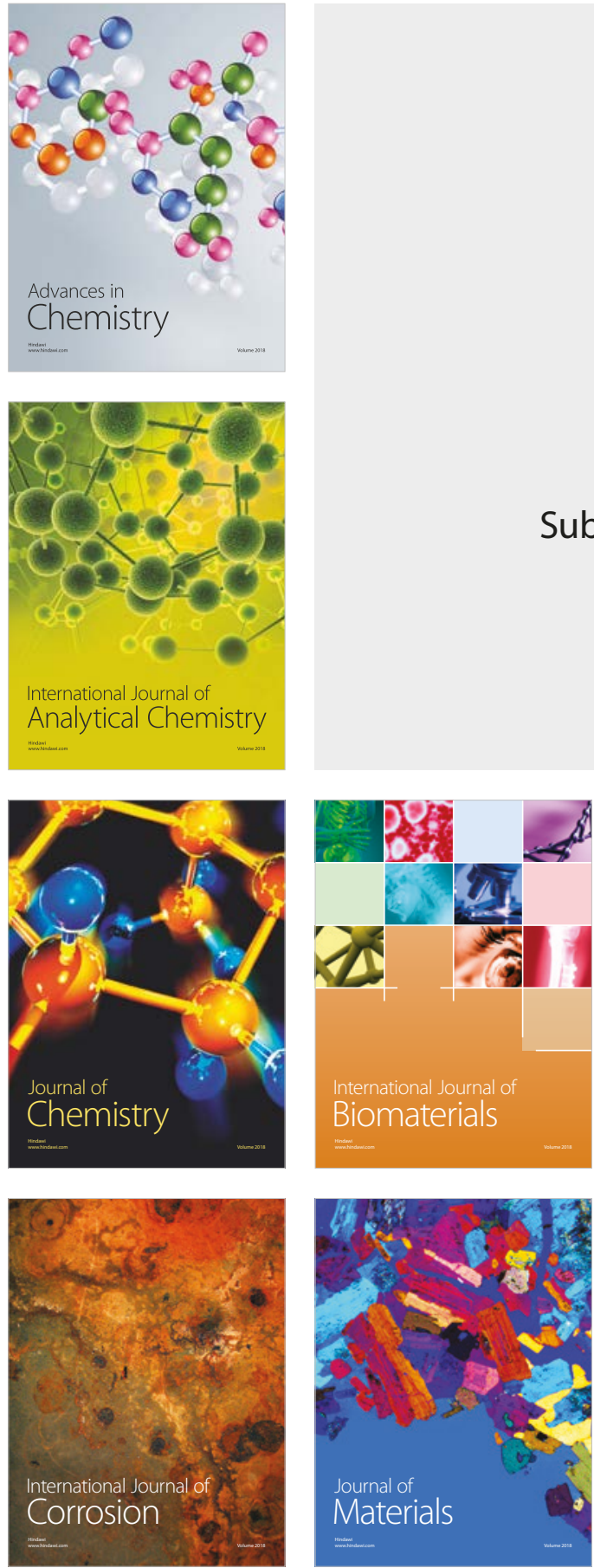

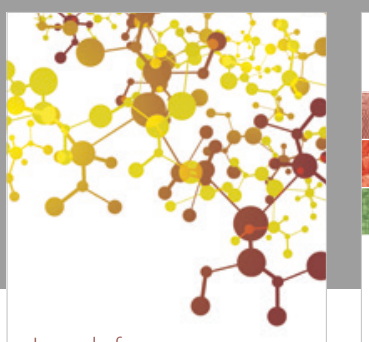

Journal of

Applied Chemistry
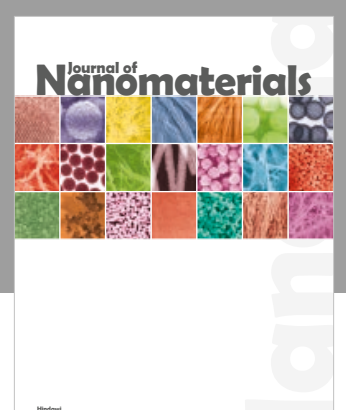

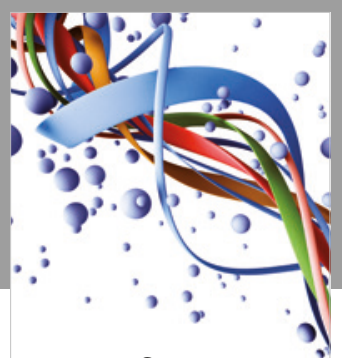

Scientifica

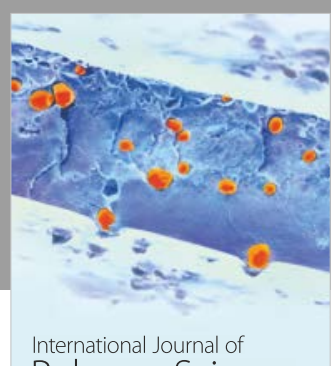

Polymer Science

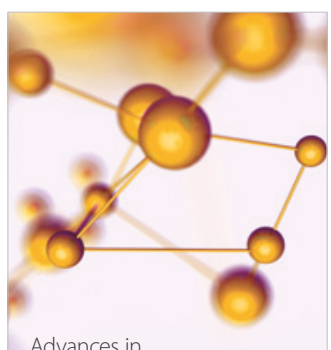

Physical Chemistry
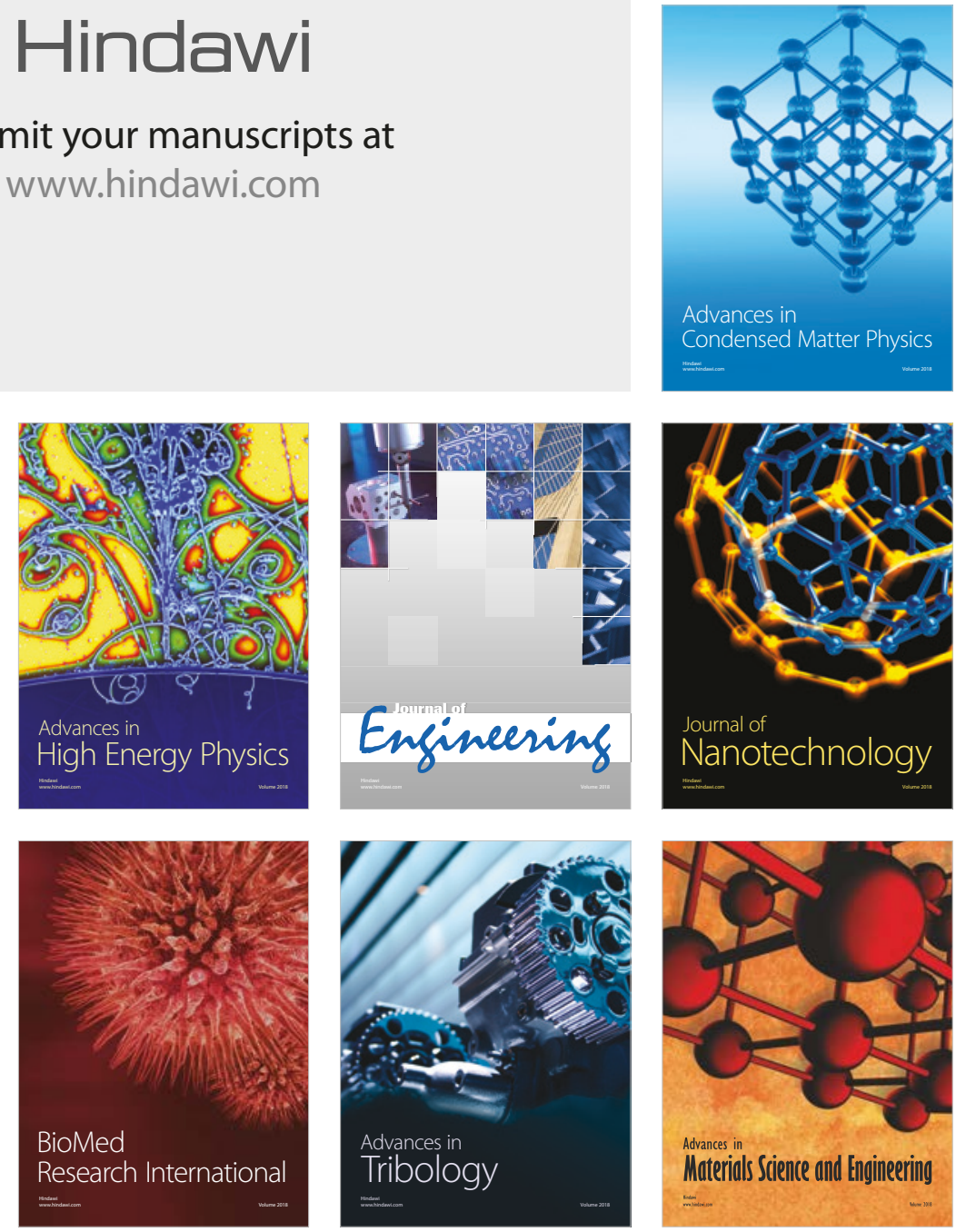